\title{
Ensino motivador de inglês por meio de um projeto de extensão na universidade federal do Pará
}

\section{Motivating english teaching through an extension project at the federal university of Pará}

\author{
DOI: $10.46814 /$ lajdv3n4-049
}

Recebimento dos originais: 01/05/2021

Aceitação para publicação: 31/06/2021

\section{Isabelly Raiane Silva dos Santos}

Mestranda em Letras pelo Programa de Pós-graduação em Letras (PPGL) da Universidade Federal do Pará (UFPA)

Docente no Instituto Federal de Educação, Ciência e Tecnologia do Pará (IFPA)

Endereço institucional: Rodovia Ernesto Acyoli, S/N, Km 3 - Nova Colina, Altamira-PA, Brasil

E-mail: isabelly.santos@ifpa.edu.br

\section{RESUMO}

No contexto da globalização, a língua inglesa vem ganhando cada vez mais notoriedade no cenário mundial. Como base nisso, inúmeros projetos de incentivo à aprendizagem de inglês vêm sido criados no estado do Pará. Dentre eles, é possível citar o projeto de extensão da Faculdade de Letras Estrangeiras Modernas (FALEM), na Universidade Federal do Pará (UFPA): Fá Lá English Project (FLEP), vinculado ao Vale Música, um programa social de música que procura capacitar crianças e adolescentes da rede pública de ensino da região metropolitana de Belém (PA) e da Serra (ES). Os alunos formam grupos musicais, corais, bandas, orquestras, e realizam apresentações em diversos locais. O FLEP funciona como um suporte, já que graduandos da UFPA têm a oportunidade de aprimorar suas habilidades dentro da sala de aula, assim como jovens músicos são encorajados a aprender uma língua estrangeira. Desse modo, os objetivos desta pesquisa são: expor aspectos que possibilitem a compreensão do papel da autonomia e da motivação no processo de aprendizagem de línguas estrangeiras, assim como evidenciar a eficácia do FLEP para uma aprendizagem de inglês bemsucedida. O referencial teórico desta investigação de cunho qualitativo é composto por conceitos propostos por autores como Ushioda (1996), Benson (2001), Paiva (2006), Brown (2007), Dantas (2008), Dantas e Magno e Silva (2008) e Magno e Silva, Dantas, Sá e Matos e Martins (2013). Partindo desses referenciais teóricos, durante o ano de 2015, uma turma de aproximadamente 15 (quinze) alunos foi analisada. Para a consolidação desta pesquisa, foram utilizadas ferramentas para a coleta de dados, tais como desenhos, fichas de auto avaliação, provas e redações dos alunos. Tendo os docentes como os principais agentes motivadores, ao final do projeto foram observados altos níveis de motivação em grande parte dos estudantes. Assim, constatou-se que a inserção de temas culturais na metodologia de ensino tornou a participação dos alunos mais ativa, o que contribuiu em grande parte no bom andamento e sucesso das atividades aplicadas.

Palavras-chave: Ensino e aprendizagem. Língua inglesa. Projeto de extensão.

\begin{abstract}
In the context of globalization, English language is gaining more and more notoriety on the world stage. Based on this, numerous projects to encourage English learning have been created in the state of Pará. Among them, it is possible to mention the extension project of the Faculty of Modern Foreign Languages (FALEM), at the Federal University of Pará (UFPA): Fá Lá English Project (FLEP), linked
\end{abstract}


to Vale Música, a social music program that seeks to train children and teenagers from public schools in the metropolitan region of Belém (PA) and Serra (ES). Students form musical groups, choirs, bands, orchestras, and perform in different locations. FLEP works as a support, as undergraduates from UFPA have the opportunity to improve their teaching skills within the classroom, as well as young musicians are encouraged to learn a foreign language. Thus, the objectives of this research are: to expose aspects that enable the understanding of the role of autonomy and motivation in the process of learning foreign languages, and to demonstrate the effectiveness of FLEP for a successful English learning. The theoretical framework of this qualitative investigation is composed of concepts proposed by authors such as Ushioda (1996), Benson (2001), Paiva (2006), Brown (2007), Dantas (2008), Dantas and Magno e Silva (2008) and Magno e Silva, Dantas, Sá e Matos and Martins (2013). Based on these theoretical references, during 2015, a class of approximately 15 (fifteen) students was analyzed. In order to consolidate this research, data collection tools were used. Among them: drawings, selfassessment forms, tests and students' essays. With teachers as the main motivating agents, at the end of the project, high levels of motivation were observed in most students. Thus, it was found that the inclusion of cultural themes in the teaching methodology made the participation of students more active, which largely contributed to the smooth running and success of the applied activities.

Keywords: Teaching and learning. English language. Extension project.

\section{INTRODUÇÃO}

Autonomia e motivação são dois pontos-chave para compreensão do processo de aprendizagem. Motivação pode ser conceituada como um estímulo consciente que encoraja o aprendente e agir. Magno e Silva, Dantas, Sá e Matos e Martins (2013, p. 62) estabelecem a motivação como "um processo dinâmico e flutuante que acompanha a trajetória de estudos do aprendente e que influencia a tomada de suas decisões e a execução das mesmas". Isso implica que o indivíduo parte de uma meta a ser alcançada para dar início ao processo de aprendizagem.

Tratando-se de autonomia, entende-se por autônomo o aluno que assume a responsabilidade por sua própria aprendizagem - diferente daquele que se auto instrui, excluindo professores para ensinar o conteúdo. No caso de um aluno autônomo, esse não depende do docente para lhe trazer conhecimento. Autonomia compreende, então, a partilha do controle de aprendizagem entre estudante e professor. A partir disso, Dantas (2008, p. 21) afirma que

\footnotetext{
"a autonomia envolve o controle compartilhado entre o professor e os alunos sobre as diversas fases de aprendizagem, desde a escolha de temas e atividades até a escolha dos critérios de avaliação. Quando o professor não impõe suas escolhas, mas ouve e acolhe a dos alunos, podese dizer que padrões de controle compartilhado estão sendo implementados."
}

Sendo a motivação outro ponto relevante para a condução desta investigação, em relação a isso, é possível observar uma relação de interdependência entre este conceito e o conceito de autonomia. Desse modo, "autonomia e motivação são uma via de mão dupla rumo ao sucesso na aprendizagem" (DANTAS; MAGNO E SILVA, 2008, p. 150). Quanto mais motivado é o aprendente, torna-se mais 
evidente os traços de autonomia. Assim, suas chances de obter êxito no processo de aprendizagem se elevam.

Partindo do princípio de que esses são construtos relevantes para a área do ensino e aprendizagem de línguas estrangeiras, fez-se pertinente um estudo envolvento o projeto de extensão Fá Lá English Project (FLEP), vinculado à Faculdade de Letras Estrangeiras Modernas (FALEM), na Universidade Federal do Pará (UFPA). A partir disso, foi válido mensurar o impacto deste projeto nos aprendentes vinculados a ele, bem como analisar o aproveitamento dos alunos expostos à língua inglesa.

Este estudo possui como objetivo geral expor aspectos que possibilitem a compreensão do papel da autonomia e da motivação no processo de aprendizagem de línguas estrangeiras. Como objetivo específico, a pesquisa busca evidenciar a eficácia do FLEP para uma aprendizagem de inglês bemsucedida.

Tendo em vista os objetivos relatados no parágrafo anterior, as perguntas de pesquisa que guiaram essa investigação foram: 1) Como são compreendidos o papel da autonomia e da motivação no processo de aprendizagem de línguas estrangeiras? 2) Qual é a eficácia do FLEP para uma aprendizagem de inglês bem-sucedida?

Na próxima seção deste artigo, a fundamentação teórica é abordada. Em seguida, a metodologia adotada durante o processo de investigação é relatada. Posteriormente, são discutidos os resultados da análise de dados e algumas de suas implicações para o ensino e aprendizagem da língua inglesa. Posteriormente, a conclusão é apresentada. Ao final, as referências bibliográficas consultadas são listadas.

\section{FUNDAMENTAÇÃO TEÓRICA}

Nesta seção, o foco volta-se para os dois construtos relevantes para o sucesso do processo de aprendizagem de uma língua estrangeira. A seguir, na primeira subseção, o conceito de autonomia é explorado. Na subseção seguinte, o conceito de motivação é abordado.

\subsection{AUTONOMIA}

De maneira contrária ao ambiente de aprendizagem da língua materna, alunos que aprendem línguas estrangeiras nem sempre terão contato com o idioma fora da sala de aula, a menos que tentem praticar o que aprenderam no ambiente formal de ensino. Nesse contexto, é necessário que os discentes sejam alunos autônomos os quais adotam estratégias de aprendizagem que facilitem o estabelecimento do processo cognitivo. 
Em relação a isso, Holec (1981) estabelece que a autonomia é a habilidade que o aprendente possui de se tornar responsável por sua própria aprendizagem. O autor acrescenta que a autonomia descreve a capacidade potencial de agir em uma situação (HOLEC, 1981).

Nesse contexto, embora o trabalho de Holec tenha sido desenvolvido apenas com o foco em alunos adultos, a autonomia começou a ser estudada em outros ambientes de aprendizagem, e pode ser observada em indivíduos pertencentes a outras faixas etárias. Isso proporcionou a ampliação do campo de estudo da Linguística Aplicada, resultando em diferentes perspectivas e conceitos.

Seguindo uma linha de pensamento semelhante às ideias expostas, para Dickinson (1987), a autonomia pode ser descrita como uma situação na qual os aprendentes são totalmente responsáveis por todas as decisões acerca de sua aprendizagem. Dessa forma, quando um aluno se sente responsável pelo conteúdo que está prestes a aprender, ficará mais motivado para adquirir conhecimentos.

Para Little (1991), o cerne da autonomia envolve capacidade de reflexão crítica e de tomada de decisão. Assim, a autonomia não se reflete apenas na forma como o discente adquire conhecimento, mas também na maneira como ele transfere o que aprendeu para um contexto mais amplo (LITTLE, 1991).

\subsection{MOTIVAÇÃO}

É inegável a importância que a motivação assume frente ao contexto de ensino e aprendizagem de línguas estrangeiras. Desde a década de 1960, as pesquisas nessa área têm avançado, principalmente a partir do desenvolvimento de diferentes teorias acerca do assunto.

Conforme estabelecido por Williams e Burden (1997), o conceito de motivação pode ser definido como um estado de alerta cognitivo e emocional o qual busca estimular a decisão consciente de agir. Entretanto, nem sempre a moticação resulta no alcance de uma meta previamente determinada.

Ainda em relação a isso, a motivação também pode ser definida como a mudança estabelecida por uma pessoa ao iniciar, orientar, coordenar, expandir, resumir e avaliar os processos cognitivos e motores (DÖRNYEI; OTTÓ, 1998). Nesse sentido, a motivação pode ser dividida em dois tipo. Assim, Dantas (2008, p. 36) explica que

um tema bastante recorrente no que tange à motivação é a distinção entre motivação extrínseca e intrínseca. Uma diferenciação operacional entre os dois tipos de motivação pode ser explicitada pelo fato de que enquanto a motivação extrínseca é causada por fatores externos como um prêmio, uma aprovação ou uma possibilidade de emprego, a motivação intrínseca vem de dentro do indivíduo e se consubstancia na satisfação pessoal em aprender.

Ainda de acordo com Dantas (2008, p. 35), “a determinação dos objetivos é um pré-requisito para o surgimento da motivação e [...] o esforço para manter a motivação é necessário a fim de que 
esses objetivos sejam atingidos. Nesse sentido, a autora relata que "o nível de motivação irá depender justamente do valor estipulado pelo sujeito para o objetivo que ele se propõe a alcançar" (DANTAS, 2008, p. 35).

\section{METODOLOGIA}

Esta pesquisa possui caráter qualitativo interpretativo e foi orientada segundo a necessidade de investigar como os alunos desmonstraram estar motivados durante as aulas do FLEP e se os professores atingiram o objetivo de sensibilizá-los a aprender inglês.

Para a consolidação deste estudo, foram utilizadas ferramentas para a coleta de dados: desenhos, fichas de auto avaliação, provas e redações dos alunos, fotos, vídeos e caderno de anotações.

Esta pesquisa foi desenvolvida a partir da análise do projeto FLEP, no qual foram ministradas aulas por discentes do curso de graduação em Licenciatura em Letras com habilitação em Inglês da UFPA aos alunos de escola pública participantes do Projeto Vale Música.

Foram formadas duas turmas de, aproximadamente, 15 (quinze) alunos em cada. Os discentes possuíam faixa etária entre 10 (dez) e 13 (treze) anos, e contaram com a orientação de 8 (oito) professores em formação os quais ministraram aulas de inglês ao longo da execução do FLEP.

As aulas ocorriam semanalmente, aos sábados, no período matutino, e tinham a duração de 1 (uma) hora. O período de aulas estendeu-se de março a novembro de 2015. O nível de proficiências dos estudantes era básico, e os encontros não contavam com a utilização de livro didático, já que todos os materias, como apostilas, provas, exercícios, flashcards e apresentações de slide em PowerPoint, eram desenvolvidos pelos próprios professores.

\section{RESULTADOS E DISCUSSÃO}

No FLEP, além de participarem das atividades em sala de aula desenvolvidas pelos professores em formação na sede no Projeto Vale Música, em Belém, os alunos contavam com aulas práticas com passeios ao ar livre e auxílio de estrangeiros - bolsistas norte-americanos disponibilizados pela UFPA para atuarem no projeto.

Ademais, as turmas de inglês formadas a partir da seleção de alguns dos jovens músicos participantes do Projeto Vale Música possuíam limite de vagas. Isso facilitou o atendimento dos professores aos alunos de maneira mais individualizada.

Nesse contexto, ao longo das aulas de inglês, a maioria dos alunos mostrou-se motivada e interessada em estudar a língua inglesa. Como estudantes de música, tornou-se sonho de muitos viajar para o exterior a fim de aprimororar e consolidar a carreira musical. Nesse sentido, o domínio do inglês seria um ponto facilitador na concretização dessa aspiração discente. 
De acordo com Brown (2007, p. 88), “comportamentos motivados extrínsecamente são mantidos em antecipação a uma recompensa externa e além do próprio indivíduo". Ou seja, o fato de os alunos traçarem como meta viajar para o exterior por meio de sua futura profissão os manteve motivados a estudar e a adquirir fluência no idioma estrangeiro.

Por intermédio dos materiais analisados (desenhos, fichas de auto avaliação, provas, redações e caderno de anotações), foi possível constatar que muitos discentes tornaram-se alunos autônomos, apresentando interesse extra sala de aula e buscando contato com o inglês sem o auxílio dos professores. Nesse sentido, Benson (2001) defende a ideia de que autonomia implica no controle da própria aprendizagem. Seguindo a mesma linha de raciocínio, Paiva (2006, p. 88) expõe que

autonomia é um sistema sócio-cognitivo complexo, que se manifesta em diferentes graus de independência e controle sobre o próprio processo de aprendizagem, envolvendo capacidades, habilidade, atividade, desejo, tomadas de decisão, escolhas e avaliação tanto como aprendiz de língua ou como seu usuário, dentro ou fora da sala de aula.

A autonomia dos alunos do FLEP pôde ser notada, por exemplo, quando esses traziam para a sala de aula questionamentos e observações a respeito de vocabulários os quais não haviam sido ensinados pelos professores. Atitudes como essa evidenciam a influência das aulas no desencandeamento de ações independentes. Nesse sentido, é evidenciado o caso de um aluno motivado que buscou aprender mais por interesse próprio.

Apesar de a maioria dos aprendentes ter apresentado resultados positivos, alguns alunos mantiveram-se indiferente às aulas. Isso acontece pois os sistemas de aprendizagem, por serem sistemas adaptativos complexos, apresentam não semelhança, não linearidade e imprevisibilidade, fazendo com que os sistemas sigam trajetórias distintas (LARSEN-FREEMAN; CAMERON, 2008). Por isso, Paiva (2006) relata que o grau de autonomia dos aprendentes também é diferente.

Além do exposto no parágrafo anterior, os professores em formação que atuaram no FLEP precisaram lidar com dificuldades, tais como: a baixa carga horária e frequência de aulas, o grande número de discentes faltosos, o que impediu o trabalho contínuo dos docentes, e a precária estrutura da sala de aula destinada aos encontros, visto que o local não possuía assentos suficientes para todos os estudantes.

De qualquer modo, tendo os docentes como os principais agentes motivadores, ao final do projeto, foram observados altos níveis de motivação e autonomia em grande parte dos alunos. Em outras palavras, o aluno foi motivado a tomar controle de seu processo cognitivo, e, por isso, foi capaz de buscar ferramentas além do ambiente da sala de aula para manter contato com a língua inglesa, como o uso de aplicativos de aprendizagem e de jogos de video game. 
Nesse sentido, para que a motivação acompanhe as mudanças do processo de aprendizagem, aprendentes precisam desenvolver habilidades e estratégias para mantê-los focados. Isso pode implicar em estabelecer objetivos a curto prazo, procurar manter-se auto motivado, motivar-se por meio de incentivos, como recompensas, ou organizar o tempo de maneira eficiente a fim de conciliar os estudos com as demais atividades (USHIODA, 2008).

\title{
5 CONCLUSÃO
}

Ushioda (1996) postula que, para manter um aluno motivado e estimular um comportamento autônomo, a aprendizagem de língua estrangeira deve atender suas necessidades, e as atividades desenvolvidas em sala de aula devem ser relevantes em suas vidas. Assim sendo,

\begin{abstract}
os aprendentes irão perceber que a língua estrangeira não é meramente um objeto de estudo abstrato ou um sistema de regras, mas sim um meio de expandir seu próprio comportamento no que tange ao modo como eles interagem com o mundo, permitindo que atendam suas próprias paixões e interesses, como acessam novas fontes de informação e materiais e como se apropriam de novas ideias e experiências (USHIODA, 1996, p. 43-44)1 ${ }^{1}$.
\end{abstract}

Como relatado, os alunos do FLEP gerenciaram sua aprendizagem a partir do momento em que buscaram estratégias de uso da língua inglesa sem intermédio dos professores. Apesar de ainda não terem garantido o alcance de suas metas, pois ainda são músicos em formação, ao final das aulas, os aprendentes estavam focados em seus interesses, dentre eles, comunicar-se, em inglês, no exterior.

Ainda, tornou-se evidente que a aprendizagem pode ser mais bem-sucedida quando o aprendente passa a compreender o funcionamento do processo cognitivo e elege estratégias que fortaleçam seu próprio estilo de aprendizagem.

\footnotetext{
${ }^{1}$ Minha tradução do original: "Students will quickly perceive that the foreign language is not merely an abstract object of study or a system of rules, but a further means of expanding their own behavioural repertoire in terms of how they interact with the world, indulge their own passions and interests, access new sources of information and materials, appropriate new ideas and experiences" (USHIODA, 1996, p. 43-44).
} 


\section{REFERÊNCIAS}

BENSON, P. Teaching and Researching Autonomy in Language Learning. Harlow: Pearson, 2001.

BROWN, J. Teaching by principles: An interactive approach to language pedagogy. Englewoods Cliffs: Prentice Hall Regents, 2007.

DANTAS, L. Gêneros textuais acadêmicos e ensino da língua inglesa: um caminho para a motivação e a autonomia. 2008. 174 f. Dissertação (Mestrado em Estudos Linguísticos) Universidade Federal do Pará, 2008.

DANTAS, L.; MAGNO E SILVA, W. Motivação e autonomia para a formação de um novo aprendente e de um novo professor. In: ASSIS, R. (Org.). Estudo da língua portuguesa e de todas as línguas que fazem a nossa. Belém: Editora Unama, p. 139-151, 2008.

DICKINSON, L. Self-instruction in language learning. Cambridge: Cambridge University Press, 1987.

DÖRNYEI, Z.; OTTO, I. Motivation in action: A process model of L2 motivation. Working papers in Applied Linguistics. Thames Valley University, London, v. 4, p. 43-69, 1998.

HOLEC, H. Autonomy and foreign language learning. Oxford: Pergamon, 1981.

LARSEN-FREEMAN, D.; CAMERON, L. Complex Systems and Applied Linguistics. Oxford: Oxford University Press, 2008.

LITTLE, D. Learner Autonomy: Definitions, Issues and Problems. Dublin: Authentik, 1991.

MAGNO E SILVA, W.; DANTAS, L.; SÁ E MATOS.; MARTINS, M. Aconselhamento linguageiro no processo de aprendizagem de inglês. Trab. linguist. Apl., Campinas, v. 52, n. 1, p. 53-72, 2013.

PAIVA, V. Autonomia e complexidade. Linguagem \& Ensino, Pelotas, v. 9, n. 1, p. 77-127, 2006.

USHIODA, E. Learner autonomy: the role of motivation. Dublin: Authentik, 1996.

USHIODA, E. Motivation and good language learners. In: GRIFFITHS, C. (Ed.). Lessons from Good Language Learners. Cambridge: Cambridge University Press, p. 19-34, 2008.

WILLIAMS, M.; BURDEN, R. L. Psychology for Language Teachers: a social constructivist approach. Cambridge: Cambridge University Press, 1997. 